ORIGINAL RESEARCH

\title{
Neutrophil-to-lymphocyte ratio for predicting palliative chemotherapy outcomes in advanced pancreatic cancer patients
}

\author{
Peng Xue ${ }^{1,2}$, Masashi Kanai ${ }^{1}$, Yukiko Mori ${ }^{3}$, Takafumi Nishimura ${ }^{3}$, Norimitsu Uza ${ }^{4}$, Yuzo Kodama ${ }^{4}$, \\ Yoshiya Kawaguchi ${ }^{5}$, Kyoichi Takaori ${ }^{5}$, Shigemi Matsumoto ${ }^{1}$, Shinji Uemoto ${ }^{5}$ \& Tsutomu Chiba ${ }^{4}$ \\ ${ }^{1}$ Department of Clinical Oncology and Pharmacogenomics, Kyoto University Graduate School of Medicine, Kyoto, Japan \\ ${ }^{2}$ Department of Medical Oncology and Shanghai Key Laboratory for Pancreatic Diseases, Shanghai First People's Hospital, Shanghai Jiaotong \\ University, Shanghai, China \\ ${ }^{3}$ Department of Translational Clinical Oncology, Kyoto University Graduate School of Medicine, Kyoto, Japan \\ ${ }^{4}$ Department of Gastroenterology and Hepatology, Kyoto University Graduate School of Medicine, Kyoto, Japan \\ ${ }^{5}$ Department of Surgery, Kyoto University Graduate School of Medicine, Kyoto, Japan
}

\section{Keywords}

Chemotherapy, inflammation, NLR,

pancreatic cancer, prognostic factor

\section{Correspondence}

Masashi Kanai, Kyoto University Hospital, 54 Shogoin-Kawahara-cho, Sakyo-ku, Kyoto 606-8507, Japan.

Tel: +81-75-751-4770;

Fax: +81-75-751-4772;

E-mail: kanai@kuhp.kyoto-u.ac.jp

\section{Funding Information}

This work was supported by a Japan-China Sasakawa Medical Fellowship.

Received: 6 November 2013; Revised: 4 January 2014; Accepted: 6 January 2014

Cancer Medicine 2014; 3(2): 406-415

doi: $10.1002 /$ cam4.204

\begin{abstract}
Several previous studies reported that the neutrophil-to-lymphocyte ratio (NLR) could be a promising prognostic factor for patients with cancer. We aimed to determine the prognostic value of NLR in patients with advanced pancreatic cancer (APC) following palliative chemotherapy. We retrospectively reviewed 252 consecutive APC patients receiving palliative chemotherapy between January 2006 and December 2012. We classified the patients according to the pretreatment NLR values $(\leq 5$ or $>5)$ into two groups and investigated the difference in treatment outcomes, including time to treatment failure (TTF) and overall survival (OS). A total of 212 patients had pretreatment NLR values of $\leq 5$ (group A), while 40 patients had an NLR of $>5$ (group B). TTF and OS were significantly shorter in group B than in group A (3.1 vs. 8.7 months and 6.0 vs. 12.8 months, respectively; both $P<0.01$ ). After adjustment for putative prognostic factors, including distant metastasis, status of recurrent/unresectable disease, pretreatment carbohydrate antigen 199 levels, and carcinoembryonic antigen levels using the Cox regression model, elevated pretreatment NLR remained an independent poor prognostic factor for OS (hazard ratio, 1.92; 95\% confidence interval, $1.27-2.90 ; P<0.01$ ). In addition, patients in group B whose NLR dropped to $\leq 5$ before the second cycle of chemotherapy showed longer TTF and OS compared with those whose NLR remained at $>5$. Our results support the idea that NLR can be a promising prognostic and predictive marker for APC patients receiving palliative chemotherapy.
\end{abstract}

\section{Introduction}

Pancreatic cancer is one of the most lethal malignancies worldwide [1], and most patients are diagnosed too late for curative resection. Even with curative resection, disease relapse within 2 years occurs in $>80 \%$ patients [2, 3]. Systemic gemcitabine-based chemotherapy has long been used as a standard therapy for patients with advanced pancreatic cancer (APC). However, the long-term efficiency and prognosis vary greatly among patients [4]. Therefore, it is clinically relevant to identify APC patients who are more likely to benefit from palliative chemotherapy.

Accumulating evidence supports a positive relationship between inflammation and cancer development and progression $[5,6]$. The interaction between tumor and host immune system promote tumor cell proliferation, metastasis, and also activate the inflammatory cascade in the 
host, which further deteriorates the general condition of cancer patients [6]. Several markers, including neutrophil-to-lymphocyte ratio (NLR), platelet to lymphocyte ratio (PLR), and modified Glasgow prognostic score (mGPS), have been proposed to estimate the magnitude of systemic inflammation in cancer patients [7-9]. Among these markers, a growing body of evidence supports the usefulness of NLR in predicting the prognosis of patients with cancer. Elevated NLR has reportedly been associated with poor survival following resection or chemotherapy in a variety of cancers [10-14]. In pancreatic cancer, an increasing number of studies have reported an association between elevated NLR $(>5)$ and poor prognosis [7, 15-17]. However, most studies included operable pancreatic cancer patients $[7,15,18]$, and the prognostic value of NLR in APC patients receiving palliative chemotherapy is still limited. In fact, only one study of a relatively small cohort $(n=89)$ focused on APC patients receiving chemotherapy and demonstrated that elevated NLR could predict poor survival [16]. Other studies that reported similar results analyzed the pooled data of patients who underwent surgery [17] or did not receive chemotherapy [7]. Therefore, the usefulness of NLR as a prognostic marker for APC patients following chemotherapy should be validated in another large cohort. Furthermore, it is unknown whether the evaluation of NLR kinetics can predict outcomes for APC patients following chemotherapy.

In this study, we aimed to determine whether elevated NLR could be an independent poor prognostic factor in APC patients following chemotherapy and whether the monitoring of decreased NLR before the second cycle of chemotherapy could predict better outcomes.

\section{Patients and Methods}

\section{Patients and treatment}

Using a prospective cohort database system (CyberOncology $^{\circledR}$, Cyber Laboratory Inc., Tokyo, Japan) [19] and electronic medical charts, we retrieved the clinical data of 269 consecutive patients with pathologically confirmed pancreatic ductal adenocarcinoma who received at least two cycles of palliative first-line chemotherapy at Kyoto University Hospital (Kyoto, Japan) between January 2006 and December 2012. In principle, NLR was calculated using the neutrophils and lymphocytes counts obtained on the same day of chemotherapy. If blood test was not performed on the same day of chemotherapy, we substituted the data obtained within 2 days of chemotherapy. Sixteen cases were excluded from this study because a set of NLR values before the first and second chemotherapy cycles was not available, and 252 patients were ultimately investigated. Patients who had once undergone radical resection ( $\mathrm{R} 0$ or $\mathrm{R} 1$ ) for primary tumors and developed recurrent disease were classified into the recurrent group $(n=73)$, while those who had an initial diagnosis of unresectable disease were placed into the initially unresectable group $(n=179)$. Palliative chemotherapy regimens included gemcitabine monotherapy $(n=156)$ [20], gemcitabine and S-1 combination therapy $(n=85)$ [21], S-1 monotherapy $(n=9)$ [22], and gemcitabine and erlotinib combination therapy $(n=2)$ [23]. The standard doses and regimen schedules were adjusted at the discretion of the treating physicians according to incidence of adverse events or the general condition of the individual patient. All patients provided written informed consent for the use of their clinical data in the medical records system for research. This study was approved by the Ethics Committee of Kyoto University Graduate School of Medicine (E1606).

\section{Demographic/clinical and laboratory variables}

Baseline patient characteristics, including laboratory data before the first cycle of palliative chemotherapy and the NLR values before the first and second cycles of chemotherapy, were collected for analysis. On the basis of previous studies,[24-26] continuous parameters were categorized for the convenience of prognostic analysis as follows; age ( $<65$ or $\geq 65$ years), Eastern Cooperative Oncology Group Performance Status (ECOG PS) score $(0-1$ or 2$)$, NLR $(\leq 5$ or $>5$ ), platelet to lymphocyte ratio (PLR) $(<150$ or $\geq 150)$, levels of carbohydrate antigen 199 (CA19-9, $<1000$ or $\geq 1000 \mathrm{U} / \mathrm{mL}$ ), carcinoembryonic antigen (CEA, $<5$ or $\geq 5 \mathrm{ng} / \mathrm{mL}$ ), C-reactive protein (CRP, $<0.5$ or $\geq 0.5 \mathrm{mg} / \mathrm{dL}$ ), lactate dehydrogenase ( $\mathrm{LDH},<250$ or $\geq 250 \mathrm{IU} / \mathrm{L}$ ), hemoglobin ( $<10$ or $\geq 10 \mathrm{~g} / \mathrm{dL})$, and albu$\min (<3.5$ or $\geq 3.5 \mathrm{~g} / \mathrm{dL})$.

\section{Statistical analysis}

Baseline patient characteristics were compared using the $\chi^{2}$ test or Fisher's exact test for dichotomous variables or the Mann-Whitney $U$ test for continuous variables. The time to treatment failure (TTF) was calculated from the date of palliative chemotherapy initiation and terminated on the date of palliative chemotherapy discontinuation for various reasons, including treatment toxicity, disease progression, or patient withdrawal. Overall survival (OS) was calculated from the date of palliative chemotherapy initiation and terminated on the date of death for any reason or censored on the last follow-up visit. TTF and OS were estimated using the Kaplan-Meier method, and differences were compared using log-rank tests. Cox 
Table 1. Baseline characteristics.

\begin{tabular}{|c|c|c|c|c|}
\hline Variables & $\begin{array}{l}\text { Total } \\
(n=252)\end{array}$ & $\begin{array}{l}\text { NLR } \leq 5 \\
(n=212)\end{array}$ & $\begin{array}{l}\mathrm{NLR}>5 \\
(n=40)\end{array}$ & $P$-value \\
\hline \multicolumn{5}{|l|}{ Age } \\
\hline$\geq 65$ & $148(58.7 \%)$ & $122(57.5 \%)$ & $26(65.0 \%)$ & \multirow[t]{2}{*}{0.48} \\
\hline$<65$ & $104(41.3 \%)$ & $90(42.5 \%)$ & $14(35.0 \%)$ & \\
\hline \multicolumn{5}{|l|}{ Gender } \\
\hline Male & $133(52.8 \%)$ & $110(51.9 \%)$ & $23(57.5 \%)$ & \multirow[t]{2}{*}{0.61} \\
\hline Female & $119(47.2 \%)$ & $102(48.1 \%)$ & $17(42.5 \%)$ & \\
\hline \multicolumn{5}{|l|}{ PS score } \\
\hline $0-1$ & $242(96.0 \%)$ & $204(96.2 \%)$ & $38(95.0 \%)$ & \multirow[t]{2}{*}{0.66} \\
\hline 2 & $10(4.0 \%)$ & $8(3.8 \%)$ & $2(5.0 \%)$ & \\
\hline \multicolumn{5}{|l|}{ Distant metastasis } \\
\hline Yes & $184(73.0 \%)$ & $152(71.7 \%)$ & $32(80.0 \%)$ & \multirow[t]{2}{*}{0.34} \\
\hline No & $68(27.0 \%)$ & $60(28.3 \%)$ & $8(20.0 \%)$ & \\
\hline \multicolumn{5}{|l|}{ Primary tumor location } \\
\hline Head & $146(57.9 \%)$ & $127(59.9 \%)$ & $19(47.5 \%)$ & \multirow[t]{2}{*}{0.16} \\
\hline Body and tail & $106(42.1 \%)$ & $85(40.1 \%)$ & $21(52.5 \%)$ & \\
\hline \multicolumn{5}{|c|}{ The status of recurrent or unresectable } \\
\hline Recurrent & $73(29.0 \%)$ & $64(30.2 \%)$ & $9(22.5 \%)$ & \multirow[t]{2}{*}{0.45} \\
\hline Unresectable & $179(71.0 \%)$ & $148(69.8 \%)$ & $31(77.5 \%)$ & \\
\hline \multicolumn{5}{|l|}{ Palliative first line } \\
\hline Gemcitabine monotherapy & $156(61.9 \%)$ & $130(61.3 \%)$ & $26(65.0 \%)$ & \multirow[t]{4}{*}{0.82} \\
\hline Gemcitabine and S-1 & $85(33.7 \%)$ & $73(34.4 \%)$ & $12(30.0 \%)$ & \\
\hline S-1 monotherapy & $9(3.6 \%)$ & $7(3.3 \%)$ & $2(5.0 \%)$ & \\
\hline Gemcitabine and Erlotinib & $2(0.8 \%)$ & $2(1.0 \%)$ & 0 & \\
\hline \multicolumn{5}{|l|}{ CA19-9 (U/mL) } \\
\hline$<1000$ & $196(77.8 \%)$ & $170(80.2 \%)$ & $26(65.0 \%)$ & \multirow[t]{2}{*}{0.04} \\
\hline$\geq 1000$ & $56(22.2 \%)$ & $42(19.8 \%)$ & $14(35.0 \%)$ & \\
\hline \multicolumn{5}{|l|}{ CEA $(n g / m L)$} \\
\hline$<5$ & $145(57.5 \%)$ & $126(59.4 \%)$ & $19(47.5 \%)$ & \multirow[t]{2}{*}{0.17} \\
\hline$\geq 5$ & $107(42.5 \%)$ & $86(40.6 \%)$ & $21(52.5 \%)$ & \\
\hline $\operatorname{CRP}(\mathrm{mg} / \mathrm{dL})$ & & & & \\
\hline$<0.5$ & $175(69.4 \%)$ & $159(75.0 \%)$ & $16(40.0 \%)$ & $<0.01$ \\
\hline$\geq 0.5$ & $77(30.6 \%)$ & $53(25.0 \%)$ & $24(60.0 \%)$ & \\
\hline LDH (IU/L) & & & & \\
\hline$<250$ & $219(86.9 \%)$ & $190(89.6 \%)$ & $29(72.5 \%)$ & 0.01 \\
\hline$\geq 250$ & $33(13.1 \%)$ & $22(10.4 \%)$ & $11(27.5 \%)$ & \\
\hline Hemoglobin (g/dL) & & & & \\
\hline$<10$ & $26(10.3 \%)$ & $20(9.4 \%)$ & $6(15.0 \%)$ & 0.27 \\
\hline$\geq 10$ & $226(89.7 \%)$ & $192(90.6 \%)$ & $34(85.0 \%)$ & \\
\hline Albumin (g/dL) & & & & \\
\hline$\geq 3.5$ & $183(72.6 \%)$ & $157(74.1 \%)$ & $26(65.0 \%)$ & 0.25 \\
\hline$<3.5$ & $69(27.4 \%)$ & 55 (25.9\%) & $14(35.0 \%)$ & \\
\hline PLR & & & & \\
\hline$\geq 150$ & $148(58.7 \%)$ & $110(51.9 \%)$ & $38(95.0 \%)$ & $<0.01$ \\
\hline$<150$ & $104(41.3 \%)$ & $102(48.1 \%)$ & $2(5.0 \%)$ & \\
\hline $\mathrm{TB}(\mathrm{mg} / \mathrm{dL})$ & & & & \\
\hline Median & 0.7 & 0.7 & 0.7 & 0.87 \\
\hline Range & $0.2-15.9$ & $0.2-15.9$ & $0.3-6.2$ & \\
\hline AST (IU/L) & & & & \\
\hline Median & 24 & 24 & 25 & 0.83 \\
\hline Range & $11-466$ & $11.00-466.00$ & $11-122$ & \\
\hline ALT (IU/L) & & & & \\
\hline Median & 24 & 25 & 24 & 0.99 \\
\hline Range & $7-564$ & $8-564$ & $7-250$ & \\
\hline Creatinin $(\mathrm{mg} / \mathrm{dL})$ & & & & \\
\hline Median & 0.7 & 0.7 & 0.7 & 0.34 \\
\hline Range & $0.2-3.2$ & $0.2-3.2$ & $0.4-1.2$ & \\
\hline
\end{tabular}


regression models were used to identify prognostic factors for TTF and OS. Prognostic factors shown to be significant in the univariate analysis were tested via multivariate analysis. The hazard ratio (HR) and 95\% confidence interval (CI) were calculated using Cox regression models. A two-tailed $P$ value of $<0.05$ was considered statistically significant. All statistical analyses were performed using SPSS statistical software (version 17.0; SPSS Inc., Chicago, IL).

\section{Results}

\section{Patient characteristics}

Patient characteristics were stratified by the pretreatment NLR values $(\leq 5$ or $>5)$ and are summarized in Table 1 . A total of 212 patients had a pretreatment NLR of $\leq 5$, while 40 had an NLR of $>5$. Most baseline characteristics were comparable between the two groups. However, the

Table 2. Univariate and multivariate analysis of poor prognostic factors for TTF.

\begin{tabular}{|c|c|c|c|c|c|c|c|c|}
\hline & \multirow[b]{2}{*}{$n$} & \multirow{2}{*}{$\begin{array}{l}\text { Median TTF } \\
\text { (95\% CI) } \\
\text { (months) }\end{array}$} & \multicolumn{3}{|c|}{ Univariate analysis } & \multicolumn{3}{|c|}{ Multivariate analysis } \\
\hline & & & $\begin{array}{l}\text { Hazard } \\
\text { ratio }\end{array}$ & $95 \% \mathrm{Cl}$ & $P$-value & $\begin{array}{l}\text { Hazard } \\
\text { ratio }\end{array}$ & $95 \% \mathrm{Cl}$ & $P$-value \\
\hline \multicolumn{9}{|l|}{ Age (years) } \\
\hline$\geq 65$ & 148 & $7.7(6.0-9.4)$ & 1 & $0.74-1.27$ & 0.83 & & & \\
\hline$<65$ & 104 & $8.0(6.5-9.5)$ & 0.97 & & & & & \\
\hline \multicolumn{9}{|l|}{ Gender } \\
\hline Female & 119 & $6.6(5.0-8.2)$ & 1 & $0.80-1.36$ & 0.77 & & & \\
\hline Male & 133 & $8.0(6.4-9.6)$ & 1.04 & & & & & \\
\hline \multicolumn{9}{|l|}{ ECOG PS } \\
\hline $0-1$ & 242 & $7.4(6.1-8.7)$ & 1 & $1.03-3.68$ & 0.04 & 1 & $0.84-3.15$ & 0.15 \\
\hline 2 & 10 & $2.2(0.0-6.4)$ & 1.95 & & & 1.62 & & \\
\hline \multicolumn{9}{|l|}{ Distant metastasis } \\
\hline No & 68 & $9.0(6.6-11.4)$ & 1 & $1.28-2.44$ & $<0.01$ & 1 & $1.12-2.18$ & $<0.01$ \\
\hline Yes & 184 & $6.9(5.8-8.0)$ & 1.77 & & & 1.56 & & \\
\hline \multicolumn{9}{|l|}{ Primary tumor location } \\
\hline Head & 146 & $6.7(5.7-7.7)$ & 1 & $0.74-1.27$ & 0.84 & & & \\
\hline Body and tail & 106 & $9.3(7.1-11.5)$ & 0.97 & & & & & \\
\hline \multicolumn{9}{|c|}{ The status of initially unresectable/recurrent } \\
\hline Recurrent & 73 & $11.9(7.2-16.6)$ & 1 & $1.34-2.46$ & $<0.01$ & 1 & $1.17-2.20$ & $<0.01$ \\
\hline Initially unresectable & 179 & $6.3(4.9-7.7)$ & 1.81 & & & 1.60 & & \\
\hline \multicolumn{9}{|l|}{ NLR } \\
\hline$\leq 5$ & 212 & $8.7(7.2-10.2)$ & 1 & $1.33-2.75$ & $<0.01$ & 1 & $1.08-2.31$ & 0.02 \\
\hline$>5$ & 40 & $3.1(2.7-3.5)$ & 1.91 & & & 1.58 & & \\
\hline \multicolumn{9}{|l|}{ PLR } \\
\hline$\leq 150$ & 104 & $9.6(6.8-12.4)$ & 1 & $0.93-1.59$ & 0.15 & & & \\
\hline$\geq 150$ & 148 & $6.3(4.9-7.7)$ & 1.22 & & & & & \\
\hline \multicolumn{9}{|l|}{ CA19-9 (U/mL) } \\
\hline$<1000$ & 196 & $8.8(7.2-10.4)$ & 1 & $1.60-3.00$ & $<0.01$ & 1 & $1.10-2.21$ & 0.01 \\
\hline$\geq 1000$ & 56 & $4.0(2.2-5.8)$ & 2.19 & & & 1.56 & & \\
\hline \multicolumn{9}{|l|}{ CEA (ng/mL) } \\
\hline$<5$ & 145 & $9.4(7.3-11.5)$ & 1 & $1.18-2.03$ & $<0.01$ & 1 & $0.99-1.76$ & 0.06 \\
\hline$\geq 5$ & 107 & $6.2(4.9-7.5)$ & 1.55 & & & 1.32 & & \\
\hline \multicolumn{9}{|l|}{$\mathrm{CRP}(\mathrm{mg} / \mathrm{dL})$} \\
\hline$<0.5$ & 175 & $8.8(6.9-10.7)$ & 1 & $1.40-2.47$ & $<0.01$ & 1 & $1.01-1.87$ & 0.05 \\
\hline$\geq 0.5$ & 77 & $4.4(2.8-6.0)$ & 1.86 & & & 1.37 & & \\
\hline \multicolumn{9}{|l|}{$\mathrm{LDH}(\mathrm{IU} / \mathrm{L})$} \\
\hline$\geq 250$ & 33 & $3.3(2.0-4.6)$ & 1 & $1.03-2.23$ & 0.04 & 1 & $0.89-2.00$ & 0.16 \\
\hline$<250$ & 219 & $7.9(6.4-9.4)$ & 1.51 & & & 1.34 & & \\
\hline \multicolumn{9}{|l|}{ Hemoglobin (g/dL) } \\
\hline$\geq 10$ & 226 & $7.5(6.2-8.8)$ & 1 & $0.74-1.75$ & 0.57 & & & \\
\hline$<10$ & 26 & $5.1(3.4-6.8)$ & 1.13 & & & & & \\
\hline \multicolumn{9}{|l|}{ Albumin (g/dL) } \\
\hline$\geq 3.5$ & 183 & $7.9(6.3-9.5)$ & 1 & $0.92-1.68$ & 0.15 & & & \\
\hline$<3.5$ & 69 & $5.1(2.4-7.8)$ & 1.24 & & & & & \\
\hline
\end{tabular}


Table 3. Univariate and multivariate analysis of poor prognostic factors for OS.

\begin{tabular}{|c|c|c|c|c|c|c|c|c|}
\hline & \multirow[b]{2}{*}{$n$} & \multirow{2}{*}{$\begin{array}{l}\text { Median OS } \\
\text { (95\% Cl) } \\
\text { (months) }\end{array}$} & \multicolumn{3}{|c|}{ Univariate analysis } & \multicolumn{3}{|c|}{ Multivariate analysis } \\
\hline & & & $\begin{array}{l}\text { Hazard } \\
\text { ratio }\end{array}$ & $95 \% \mathrm{Cl}$ & $P$-value & $\begin{array}{l}\text { Hazard } \\
\text { ratio }\end{array}$ & $95 \% \mathrm{Cl}$ & $P$-value \\
\hline \multicolumn{9}{|l|}{ Age (years) } \\
\hline$\geq 65$ & 148 & $12.1(10.6-13.6)$ & 1 & $0.74-1.29$ & 0.87 & & & \\
\hline$<65$ & 104 & $11.3(10.0-12.6)$ & 0.98 & & & & & \\
\hline \multicolumn{9}{|l|}{ Gender } \\
\hline Female & 119 & $11.9(10.5-13.3)$ & 1 & $0.82-1.43$ & 0.56 & & & \\
\hline Male & 133 & $11.9(10.0-13.8)$ & 1.09 & & & & & \\
\hline \multicolumn{9}{|l|}{ ECOG PS } \\
\hline $0-1$ & 242 & $12.0(10.8-13.2)$ & 1 & $1.09-3.92$ & 0.02 & 1 & $0.91-3.46$ & 0.09 \\
\hline 2 & 10 & $4.4(3.2-5.6)$ & 2.07 & & & 1.78 & & \\
\hline \multicolumn{9}{|l|}{ Distant metastasis } \\
\hline No & 68 & $16.7(11.0-22.4)$ & 1 & $1.49-2.98$ & $<0.01$ & 1 & $1.27-2.60$ & $<0.01$ \\
\hline Yes & 184 & $11.2(10.0-12.4)$ & 2.11 & & & 1.81 & & \\
\hline \multicolumn{9}{|l|}{ Primary tumor location } \\
\hline Body and tail & 106 & $12.2(10.4-14.0)$ & 1 & $0.72-1.26$ & 0.72 & & & \\
\hline Head & 146 & $11.2(9.9-12.5)$ & 0.95 & & & & & \\
\hline \multicolumn{9}{|c|}{ The status of initially unresectable/recurrent } \\
\hline Recurrent & 73 & $15.6(10.9-20.3)$ & 1 & $1.22-2.30$ & $<0.01$ & 1 & $1.08-2.12$ & 0.02 \\
\hline Initially unresectable & 179 & $11.1(9.8-12.4)$ & 1.67 & & & 1.51 & & \\
\hline \multicolumn{9}{|l|}{ NLR } \\
\hline$\leq 5$ & 212 & $12.8(10.7-14.9)$ & 1 & $1.50-3.15$ & $<0.01$ & 1 & $1.27-2.90$ & $<0.01$ \\
\hline$>5$ & 40 & $6.0(2.8-9.2)$ & 2.17 & & & 1.92 & & \\
\hline \multicolumn{9}{|l|}{ PLR } \\
\hline$\leq 150$ & 104 & $15.0(13.3-16.7)$ & 1 & $1.05-1.85$ & 0.02 & 1 & $0.79-1.49$ & 0.63 \\
\hline$\geq 150$ & 148 & $10.6(9.6-11.6)$ & 1.39 & & & 1.08 & & \\
\hline \multicolumn{9}{|l|}{ CA19-9 (U/mL) } \\
\hline$<1000$ & 196 & $13.4(11.4-15.4)$ & 1 & $1.78-3.45$ & $<0.01$ & 1 & $1.24-2.56$ & $<0.01$ \\
\hline$\geq 1000$ & 56 & $6.5(4.6-8.4)$ & 2.48 & & & 1.78 & & \\
\hline \multicolumn{9}{|l|}{ CEA $(n g / m L)$} \\
\hline$<5$ & 145 & $14.8(12.5-17.1)$ & 1 & $1.31-2.32$ & $<0.01$ & 1 & $1.11-2.04$ & 0.01 \\
\hline$\geq 5$ & 107 & $10.1(8.9-11.3)$ & 1.74 & & & 1.50 & & \\
\hline \multicolumn{9}{|l|}{$\operatorname{CRP}(\mathrm{mg} / \mathrm{dL})$} \\
\hline$<0.5$ & 175 & $13.4(11.3-15.5)$ & 1 & $1.37-2.48$ & $<0.01$ & 1 & $0.99-1.88$ & 0.06 \\
\hline$\geq 0.5$ & 77 & $7.6(4.6-10.6)$ & 1.84 & & & 1.36 & & \\
\hline \multicolumn{9}{|l|}{ LDH (IU/L) } \\
\hline$<250$ & 219 & $12.3(10.8-13.8)$ & 1 & $1.00-2.22$ & 0.05 & 1 & $0.84-1.98$ & 0.24 \\
\hline$\geq 250$ & 33 & $6.6(2.7-10.5)$ & 1.49 & & & 1.29 & & \\
\hline \multicolumn{9}{|l|}{ Hemoglobin $(\mathrm{g} / \mathrm{dL})$} \\
\hline$\geq 10$ & 226 & $12.0(10.6-13.4)$ & 1 & $0.74-1.88$ & 0.48 & & & \\
\hline$<10$ & 26 & $9.6(5.7-13.5)$ & 1.18 & & & & & \\
\hline \multicolumn{9}{|l|}{ Albumin (g/dL) } \\
\hline$\geq 3.5$ & 183 & $12.2(10.6-13.8)$ & 1 & $0.99-1.83$ & 0.06 & & & \\
\hline$<3.5$ & 69 & $10.0(6.7-13.3)$ & 1.34 & & & & & \\
\hline
\end{tabular}

following factors, including CA19-9 $(\geq 1000 \mathrm{U} / \mathrm{mL})$ levels, CRP $(\geq 0.5 \mathrm{mg} / \mathrm{dL})$ levels, LDH ( $\geq 250 \mathrm{IU} / \mathrm{L})$ levels, and PLR $(\geq 150)$ were more common in the NLR $>5$ group.

\section{Prognostic factors for poorer TTF and OS}

Univariate analysis identified eight prognostic factors associated with poorer TTF, including an ECOG PS of 2, distant metastasis, the status of unresectable disease, a pretreatment NLR of $>5$, CA19-9 levels of $\geq 1000 \mathrm{U} / \mathrm{mL}$, CEA levels of $\geq 5 \mathrm{ng} / \mathrm{mL}$, CRP levels of $\geq 0.5 \mathrm{mg} / \mathrm{dL}$, and LDH levels of $\geq 250 \mathrm{IU} / \mathrm{L}$. All these factors were subsequently analyzed in multivariate analysis. A total of five factors, including distant metastasis, status of unresectable disease, a pretreatment NLR of $>5$, CA19-9 levels of $\geq 1000 \mathrm{U} / \mathrm{mL}$, and CRP levels of $\geq 0.5 \mathrm{mg} / \mathrm{dL}$, remained 
Table 4. The NLR thresholds and relationship with survival.

\begin{tabular}{|c|c|c|c|c|c|c|c|c|c|}
\hline & & \multirow[b]{2}{*}{$n(\%)$} & \multirow{2}{*}{$\begin{array}{l}\text { Median OS } \\
(95 \% \mathrm{CI}) \\
\text { (months) }\end{array}$} & \multicolumn{3}{|c|}{ Univariate analysis } & \multicolumn{3}{|c|}{ Multivariate analysis $^{1}$} \\
\hline & & & & Hazard ratio & $95 \% \mathrm{Cl}$ & $P$-value & $\begin{array}{l}\text { Hazard } \\
\text { ratio }\end{array}$ & $95 \% \mathrm{Cl}$ & $P$-value \\
\hline \multirow[t]{10}{*}{ NLR } & $\leq 1$ & $14(5.6)$ & $12.8(9.4-16.2)$ & 1 & $0.86-3.29$ & 0.13 & 1 & $0.69-2.71$ & 0.37 \\
\hline & $>1$ & $238(94.4)$ & $11.7(10.4-13.0)$ & 1.68 & & & 1.37 & & \\
\hline & $\leq 2$ & 83 (32.9) & $14.8(11.5-18.2)$ & 1 & $1.13-2.05$ & 0.01 & 1 & $0.88-1.66$ & 0.24 \\
\hline & $>2$ & $169(67.1)$ & $10.7(9.3-12.1)$ & 1.52 & & & 1.21 & & \\
\hline & $\leq 3$ & $158(62.7)$ & $13.4(11.1-15.7)$ & 1 & $1.26-2.23$ & $<0.01$ & 1 & $1.18-2.11$ & $<0.01$ \\
\hline & $>3$ & $94(37.3)$ & $8.6(6.2-11.0)$ & 1.68 & & & 1.57 & & \\
\hline & $\leq 4$ & $194(77.0)$ & $13.3(11.4-15.2)$ & 1 & $1.44-2.78$ & $<0.01$ & 1 & $1.36-2.67$ & $<0.01$ \\
\hline & $>4$ & $58(23.0)$ & $7.3(5.6-9.0)$ & 2.00 & & & 1.91 & & \\
\hline & $\leq 5$ & $212(84.1)$ & $12.8(10.7-14.9)$ & 1 & $1.50-3.15$ & $<0.01$ & 1 & $1.49-3.15$ & $<0.01$ \\
\hline & $>5$ & $40(15.9)$ & $6.0(2.8-9.2)$ & 2.17 & & & 2.16 & & \\
\hline
\end{tabular}

${ }^{1}$ Multivariable analysis was adjusted for distant metastasis, status of recurrent, CA19-9, and CEA.

A

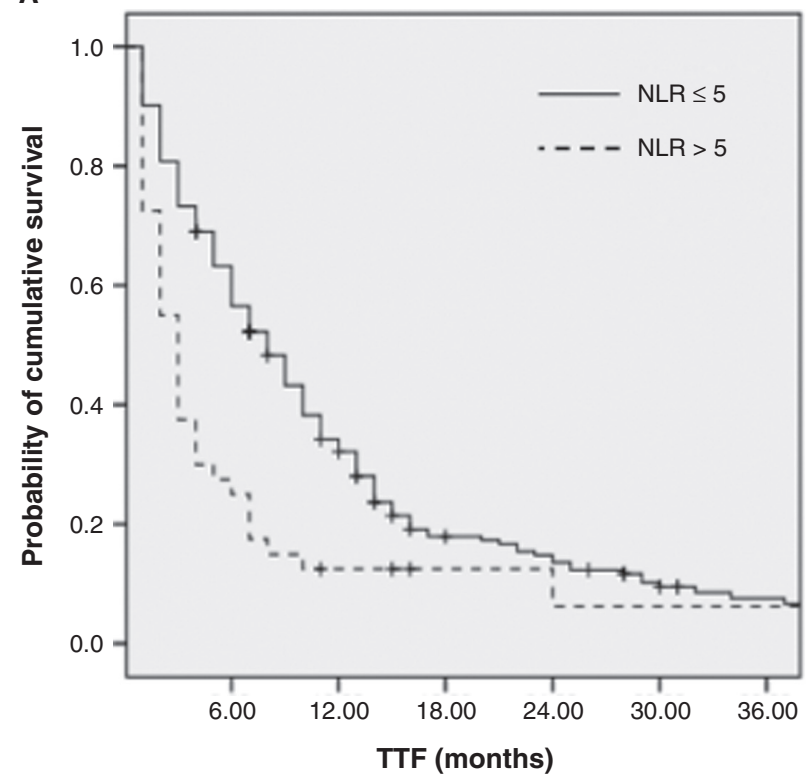

B

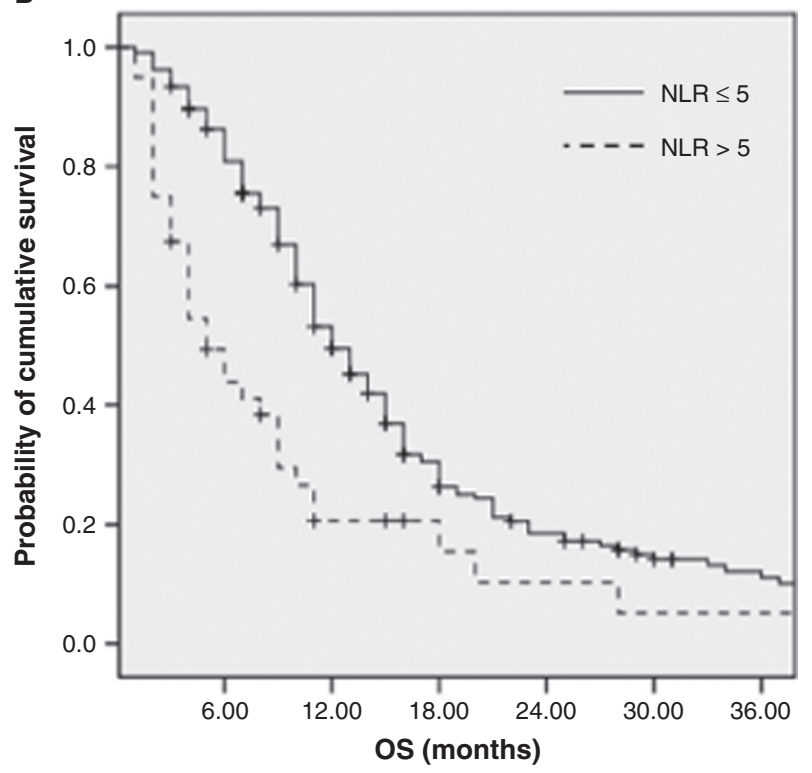

Figure 1. TTF (A) and OS (B) according to basal NLR in APC patients following palliative chemotherapy.

independent prognostic factors for poorer TTF in APC patients following chemotherapy (Table 2).

The same analysis was performed for OS, and a total of five factors, including distant metastasis, status of unresectable disease, a pretreatment NLR of $>5$, CA19-9 levels of $\geq 1000 \mathrm{U} / \mathrm{mL}$ and CEA levels of $\geq 5 \mathrm{ng} / \mathrm{mL}$, remained independent prognostic factors after multivariate analysis (Table 3).

\section{Relationship between NLR thresholds and OS}

Table 4 shows the relationship between different thresholds of NLR and OS. An NLR cutoff value of 5 could discriminate patients with poorer survival and the highest HR in our cohort.

\section{Comparison of TTF and OS stratified by pretreatment NLR}

The median TTF and OS in patients with a pretreatment NLR of $>5$ was 3.1 (95\% CI, 2.7-3.5) months and 6.0 (95\% CI, 2.8-9.2) months, respectively, which were significantly shorter compared with those of patients with an NLR of $\leq 5$ (TTF and OS, 8.7 [95\% CI, 7.2-10.2] months and 12.8 [95\% CI, 10.7-14.9] months, respectively; both $P<0.01$; Fig. $1 \mathrm{~A}$ and $\mathrm{B})$. 
A
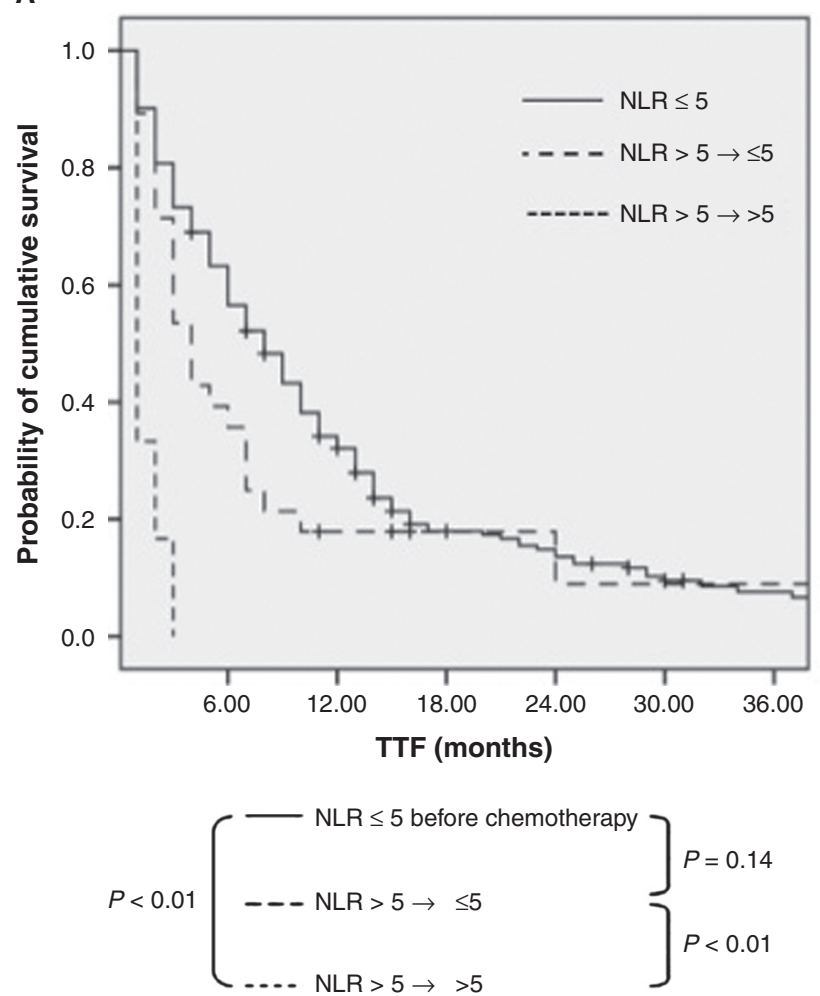

B

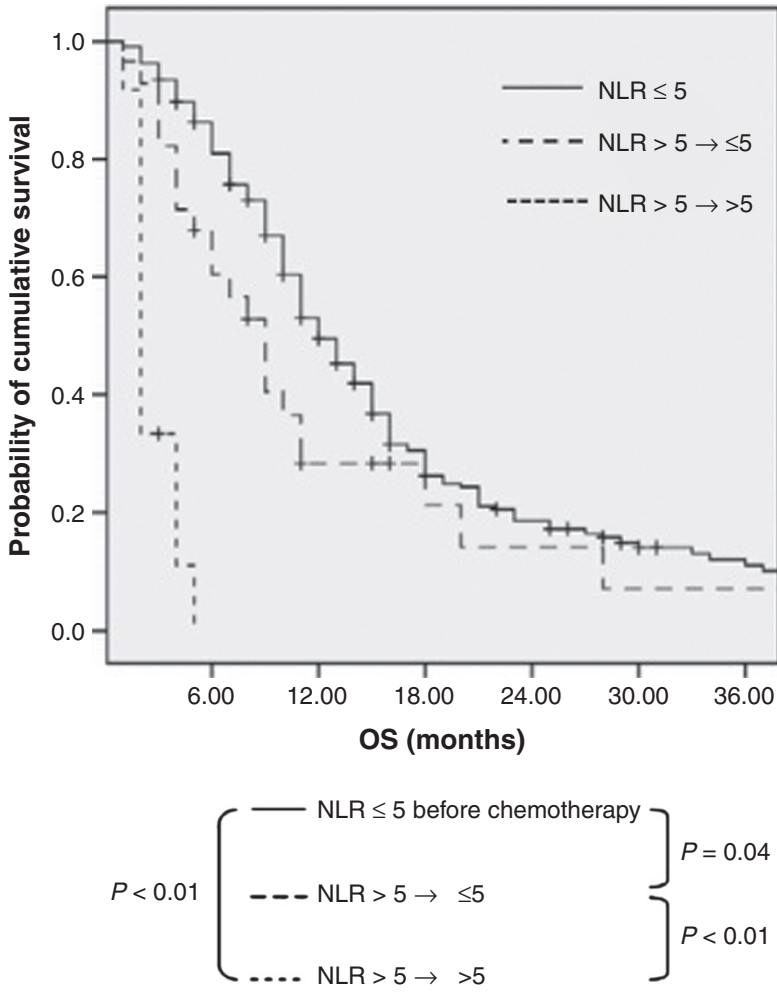

Figure 2. TTF (A) and OS (B) according to NLR change before the second cycle of chemotherapy in APC.

Table 5. Summary of published studies reporting the association between NLR and the prognosis of APC patients receiving chemotherapy.

\begin{tabular}{|c|c|c|c|c|c|}
\hline Study & Year & $n$ & $\begin{array}{l}\text { Number of patients } \\
\text { with NLR }>5(\%)\end{array}$ & $\begin{array}{l}\text { Overall survival } \\
\text { (NLR }>5 \text { vs. } \leq 5) \\
\text { (months) }\end{array}$ & $\begin{array}{l}\text { Hazard ratio } \\
(\text { NLR } \leq 5 \text { was set at } 1)\end{array}$ \\
\hline An X et al. [16] & 2010 & 89 & $16(17.9)$ & 2.4 versus 7.7 & $\mathrm{HR}=4.49, P=0.013$ \\
\hline Wang DS et al. [17] & 2012 & 86 & 12 (13.9) & 5.8 versus 10.2 & NA \\
\hline Stotz $\mathrm{M}$ et $\mathrm{al}^{1}[7]$ & 2013 & 261 & $79(30.3)$ & NA & $\mathrm{HR}=2.53, P<0.01$ \\
\hline Our study & 2013 & 253 & $40(15.8)$ & 6.0 versus 12.8 & $\mathrm{HR}=1.95, P<0.01$ \\
\hline
\end{tabular}

NA, not available.

${ }^{1}$ This study $(n=261)$ pooled the data from patients who received chemotherapy $(n=179)$ and no chemotherapy $(n=82)$.

\section{NLR drop $(\leq 5)$ before the second cycle of chemotherapy predicted favorable TTF and os}

To test whether the monitoring of the drop in NLR before the second cycle of chemotherapy could predict better outcomes, patients with a pretreatment NLR of $>5$ were categorized into two groups according to their NLR levels before the first and second cycles of chemotherapy as follows: group $1, \mathrm{NLR}>5$ at baseline and drop to $\leq 5$ before the second cycle of chemotherapy $(n=28)$; and group 2, NLR $>5$ before both the first and second cycles of chemotherapy $(n=12)$. Patients in group 1 demon- strated significantly improved TTF and OS compared with those in group 2 (4.3 vs. 1.4 months and 9.3 vs. 2.7 months, respectively; both $P<0.01$; Fig. $2 \mathrm{~A}$ and $\mathrm{B})$.

\section{Discussion}

Growing evidence supports a positive relationship between inflammation and cancer development and progression [5, 6]. NLR is attracting more and more researchers' attention because it is readily measurable in peripheral blood and is likely to reflect the magnitude of the systemic inflammatory response. An increasing number of studies have reported that elevated NLR can be a marker of poorer 
prognosis in a variety of cancers [10-14]. Elevated NLR is often accompanied by elevated neutrophil levels and relative lymphocytopenia. Elevated neutrophil levels can promote tumor cell progression by upregulating a variety of inflammatory cytokines and providing a suitable microenvironment for tumor growth [27, 28]. Furthermore, lymphocytopenia arising from numerous inhibitory immunologic mediators released by tumor cells represents an immunosuppressive condition in cancer patients and contributes to poorer outcome [29].

In this study, we aimed to determine whether elevated pretreatment NLR was associated with poorer prognosis for APC patients receiving palliative chemotherapy. Cox regression analysis identified a total of five factors, including distant metastasis, status of unresectable disease, a pretreatment NLR of $>5$, CA19-9 levels of $\geq 1000 \mathrm{U} / \mathrm{mL}$, and CEA levels of $\geq 5 \mathrm{ng} / \mathrm{mL}$, that were associated with poorer OS in our cohort. We observed significantly shorter TTF and OS among patients with a pretreatment NLR of $>5$ compared with those among patients with an NLR of $\leq 5$. The median OS was 6.0 months in patients with an NLR of $>5$ and 12.8 months in patients with an NLR of $\leq 5$. In addition, the NLR cutoff value of 5 was determined to be optimal in our cohort. Dexamethasone is commonly used for antiemetic purpose in systemic chemotherapy; however, the mean dose of dexamethasone used for antiemetic purpose was almost equal (2.2 mg) between group A and group B and it was unlikely that this affected our current results. The present results are in line with those of previous studies $[16,17]$ reporting that elevated NLR was an independent prognostic factor for OS in APC patients receiving palliative chemotherapy; these data from published studies are summarized in Table 5. The proportion of patients with a pretreatment NLR of $>5$ in existing research are comparable across studies. To the best of our knowledge, our current study comprised the largest number of APC patients who received palliative chemotherapy, and our results strongly support the hypothesis that elevated NLR $(>5)$ can be a reliable and reproducible marker for identifying a subgroup of APC patients with poorer prognosis following palliative chemotherapy.

We also demonstrated that NLR kinetics could predict treatment outcome in APC patients following palliative chemotherapy. Patients whose pretreatment NLR values of $>5$ dropped to $\leq 5$ before the second cycle of chemotherapy demonstrated significantly longer TTF and OS compared with those whose NLR values remained at $>5$ before the second cycle of chemotherapy. A total of five patients developed grade 3 or higher neutropenia during the first cycle of chemotherapy in group B. A persistent NLR of $>5$ before the second cycle of chemotherapy remained an independent poor predictive marker of TTF and OS (both $P<0.01$ ) after adjusting the incidence of grade 3 or higher neutropenia during the first cycle of chemotherapy. Persistent elevation of NLR may reflect the severe systemic inflammatory response in the body and aggressive tumor features. Our results are in line with those of the previous study by Chua et al. [11] They investigated a total of 162 patients with metastatic colorectal cancer who received palliative chemotherapy and reported that patients whose pretreatment NLR values of $>5$ dropped to $\leq 5$ before the second chemotherapy cycle demonstrated significantly longer progression-free survival and a trend toward longer OS compared with patients with a persistent NLR of $>5$. Therefore, evaluation of NLR before the second cycle of chemotherapy can help physicians to predict chemotherapy resistance and reconsider the treatment strategy at an earlier time point in daily clinical practice.

In contrast to NLR, we were unable to validate the prognostic value of PLR or mGPS in our cohort, although some researchers reported that these play prognostic roles in patients with cancer $[8,9]$. This study was limited by its retrospective design. In addition, chemotherapy regimens differed among patients; however, it is unlikely that chemotherapy regimen heterogeneity affected the current results because almost $99 \%$ patients received gemcitabine, S-1, or gemcitabine/S-1 combination therapy, and the efficacies of these three regimens were not statistically different in a large randomized phase III study [30].

In summary, our results strongly support the idea that NLR can be a promising prognostic marker for APC patients receiving palliative chemotherapy. Furthermore, evaluation of NLR before the second cycle of chemotherapy can help physicians to predict response to palliative chemotherapy at an earlier time point. Future prospective studies are warranted to verify the usefulness of monitoring NLR in treating patients with APC.

\section{Acknowledgments}

This work was supported by a Japan-China Sasakawa Medical Fellowship.

\section{Conflict of Interest}

None declared.

\section{References}

1. Siegel, R., D. Naishadham, and A. Jemal. 2013. Cancer statistics, 2013. CA Cancer J. Clin. 63:11-30.

2. Stathis, A., and M. J. Moore. 2010. Advanced pancreatic carcinoma: current treatment and future challenges. Nat. Rev. Clin. Oncol. 7:163-172. 
3. Katz, M. H., H. Wang, J. B. Fleming, C. C. Sun, R. F. Hwang, R. A. Wolff, et al. 2009. Long-term survival after multidisciplinary management of resected pancreatic adenocarcinoma. Ann. Surg. Oncol. 16:836-847.

4. Ciliberto, D., C. Botta, P. Correale, M. Rossi, M. Caraglia, P. Tassone, et al. 2013. Role of gemcitabine-based combination therapy in the management of advanced pancreatic cancer: a meta-analysis of randomised trials. Eur. J. Cancer 49:593-603.

5. Grivennikov, S. I., F. R. Greten, and M. Karin. 2010. Immunity, inflammation, and cancer. Cell 140:883-899.

6. Mantovani, A., P. Allavena, A. Sica, and F. Balkwill. 2008. Cancer-related inflammation. Nature 454:436-444.

7. Stotz, M., A. Gerger, F. Eisner, J. Szkandera, H. Loibner, A. L. Ress, et al. 2013. Increased neutrophil-lymphocyte ratio is a poor prognostic factor in patients with primary operable and inoperable pancreatic cancer. Br. J. Cancer 109:416-421.

8. Smith, R. A., L. Bosonnet, M. Raraty, R. Sutton, J. P. Neoptolemos, F. Campbell, et al. 2009. Preoperative platelet-lymphocyte ratio is an independent significant prognostic marker in resected pancreatic ductal adenocarcinoma. Am. J. Surg. 197:466-472.

9. Proctor, M. J., D. S. Morrison, D. Talwar, S. M. Balmer, D. S. O’Reilly, A. K. Foulis, et al. 2011. An inflammationbased prognostic score (mGPS) predicts cancer survival independent of tumour site: a glasgow inflammation outcome study. Br. J. Cancer 104:726-734.

10. Gomez, D., G. Morris-Stiff, G. J. Toogood, J. P. Lodge, and K. R. Prasad. 2008. Impact of systemic inflammation on outcome following resection for intrahepatic cholangiocarcinoma. J. Surg. Oncol. 97:513-518.

11. Chua, W., K. A. Charles, V. E. Baracos, and S. J. Clarke. 2011. Neutrophil/lymphocyte ratio predicts chemotherapy outcomes in patients with advanced colorectal cancer. Br. J. Cancer 104:1288-1295.

12. Azab, B., V. R. Bhatt, J. Phookan, S. Murukutla, N. Kohn, T. Terjanian, et al. 2012. Usefulness of the neutrophilto-lymphocyte ratio in predicting short- and long-term mortality in breast cancer patients. Ann. Surg. Oncol. 19:217-224.

13. Pichler, M., G. C. Hutterer, C. Stoeckigt, T. F. Chromecki, T. Stojakovic, S. Golbeck, et al. 2013. Validation of the pre-treatment neutrophil-lymphocyte ratio as a prognostic factor in a large European cohort of renal cell carcinoma patients. Br. J. Cancer 108:901907.

14. Jung, M. R., Y. K. Park, O. Jeong, J. W. Seon, S. Y. Ryu, D. Y. Kim, et al. 2011. Elevated preoperative neutrophil to lymphocyte ratio predicts poor survival following resection in late stage gastric cancer. J. Surg. Oncol. 104:504-510.

15. Garcea, G., N. Ladwa, C. P. Neal, M. S. Metcalfe, A. R. Dennison, and D. P. Berry. 2011. Preoperative neutrophil-to-lymphocyte ratio (NLR) is associated with reduced disease-free survival following curative resection of pancreatic adenocarcinoma. World J. Surg. 35:868-872.

16. An, X., P. R. Ding, Y. H. Li, F. H. Wang, Y. X. Shi, Z. Q. Wang, et al. 2010. Elevated neutrophil to lymphocyte ratio predicts survival in advanced pancreatic cancer. Biomarkers 15:516-522.

17. Wang, D. S., H. Y. Luo, M. Z. Qiu, Z. Q. Wang, D. S. Zhang, F. H. Wang, et al. 2012. Comparison of the prognostic values of various inflammation based factors in patients with pancreatic cancer. Med. Oncol. 29:30923100 .

18. Bhatti, I., O. Peacock, G. Lloyd, M. Larvin, and R. I. Hall. 2010. Preoperative hematologic markers as independent predictors of prognosis in resected pancreatic ductal adenocarcinoma: neutrophil-lymphocyte versus platelet-lymphocyte ratio. Am. J. Surg. 200:197-203.

19. Matsumoto, S., T. Nishimura, M. Kanai, H. Ishiguro, H. Yasuda, K. Yoshikawa, et al. 2007. Development of a novel information technology (IT) system using the electronic medical record (EMR) in daily clinical practice. J. Clin. Oncol. 25(Suppl.):18S (Abstr 17066).

20. Burris, H. A. 3rd, M. J. Moore, J. Andersen, M. R. Green, M. L. Rothenberg, M. R. Modiano, et al. 1997. Improvements in survival and clinical benefit with gemcitabine as first-line therapy for patients with advanced pancreas cancer: a randomized trial. J. Clin. Oncol. 15:2403-2413.

21. Nakamura, K., T. Yamaguchi, T. Ishihara, K. Sudo, H. Kato, H. Saisho, et al. 2006. Phase II trial of oral S-1 combined with gemcitabine in metastatic pancreatic cancer. Br. J. Cancer 94:1575-1579.

22. Okusaka, T., A. Funakoshi, J. Furuse, N. Boku, K. Yamao, S. Ohkawa, et al. 2008. A late phase II study of S-1 for metastatic pancreatic cancer. Cancer Chemother. Pharmacol. 61:615-621.

23. Moore, M. J., D. Goldstein, J. Hamm, A. Figer, J. R. Hecht, S. Gallinger, et al. 2007. Erlotinib plus gemcitabine compared with gemcitabine alone in patients with advanced pancreatic cancer: a phase III trial of the National Cancer Institute of Canada Clinical Trials Group. J. Clin. Oncol. 25:1960-1966.

24. Tanaka, T., M. Ikeda, T. Okusaka, H. Ueno, C. Morizane, A. Hagihara, et al. 2008. Prognostic factors in Japanese patients with advanced pancreatic cancer treated with single-agent gemcitabine as first-line therapy. Jpn. J. Clin. Oncol. 38:755-761.

25. Papadoniou, N., C. Kosmas, K. Gennatas, A. Polyzos, D. Mouratidou, E. Skopelitis, et al. 2008. Prognostic factors in patients with locally advanced (unresectable) or metastatic pancreatic adenocarcinoma: a retrospective analysis. Anticancer Res. 28:543-549.

26. Haas, M., R. P. Laubender, P. Stieber, S. Holdenrieder, C. J. Bruns, R. Wilkowski, et al. 2010. Prognostic relevance of CA 19-9, CEA, CRP, and LDH kinetics in patients 
treated with palliative second-line therapy for advanced pancreatic cancer. Tumour Biol. 31:351-357.

27. Jablonska, J., S. Leschner, K. Westphal, S. Lienenklaus, and S. Weiss. 2010. Neutrophils responsive to endogenous IFN-beta regulate tumor angiogenesis and growth in a mouse tumor model. J. Clin. Invest. 120:1151-1164.

28. Fridlender, Z. G., J. Sun, S. Kim, V. Kapoor, G. Cheng, L. Ling, et al. 2009. Polarization of tumor-associated neutrophil phenotype by TGF-beta: "N1" versus "N2" TAN. Cancer Cell 16:183-194.
29. Salazar-Onfray, F., M. N. López, and A. Mendoza-Naranjo. 2007. Paradoxical effects of cytokines in tumor immune surveillance and tumor immune escape. Cytokine Growth Factor Rev. 18:171-182.

30. Ueno, H., T. Ioka, M. Ikeda, S. Ohkawa, H. Yanagimoto, N. Boku, et al. 2013. Randomized phase III study of gemcitabine plus S-1 (GS) versus S-1 versus gemcitabine (GEM) in unresectable advanced pancreatic cancer (PC) in Japan and Taiwan: GEST study. J. Clin. Oncol. 31:16401648 . 